\title{
SCARS, MARKS AND TATTOOS (SMT): SOFT BIOMETRIC FOR SUSPECT AND VICTIM IDENTIFICATION
}

\author{
Jung-Eun Lee, Anil K. Jain and Rong Jin \\ Michigan State University \\ Computer Science and Engineering \\ East Lansing, MI 48823, USA
}

\begin{abstract}
Scars, marks and tattoos (SMT) are being increasingly used for suspect and victim identification in forensics and law enforcement agencies. Tattoos, in particular, are getting serious attention because of their visual and demographic characteristics as well as their increasing prevalence. However, current tattoo matching procedure requires human-assigned class labels in the ANSI/NIST ITL 1-2000 standard which makes it time consuming and subjective with limited retrieval performance. Further, tattoo images are complex and often contain multiple objects with large intraclass variability, making it very difficult to assign a single category in the ANSI/NIST standard. We describe a contentbased image retrieval (CBIR) system for matching and retrieving tattoo images. Based on Scale Invariant Feature Transform (SIFT) features extracted from tattoo images and optional accompanying demographical information, our system computes feature-based similarity between the query tattoo image and tattoos in the criminal database. Experimental results on two different tattoo databases show encouraging results.
\end{abstract}

\section{INTRODUCTION}

A wide variety of biometric systems have been developed for automatic recognition of individuals based on their anatomical (e.g., fingerprint, face, and iris) and behavioral (e.g., signature and gait) characteristics [1]. Among these biometric modalities, fingerprint is one of the most popular and successful traits with over one hundred years of history, particularly in law enforcement and forensics. The success of fingerprint matching in forensics has also lead to its widespread use in various government identification applications like the US-VISIT program, the Transaction Workers Identification Credentials (TWIC) program, and the Registered Traveler (RT) program [2].

Despite the tremendous progress in automatic fingerprint recognition, (see NIST Fingerprint Vendor Technology Evaluation, FpVTE, [3]), there are still situations where fingerprints alone are not able to identify a person with sufficient confidence. This is especially true when the fingerprint image quality is poor and the acquired image is only partial, as in the case of latent fingerprints. This is one of the main motivations for the Next Generation Identification (NGI) System being developed by the FBI [4]. The NGI system will utilize additional biometric modalities, such as face, palmprints, and iris to augment evidence provided by fingerprints. Another noticeable fact is that the NGI system will not only include the primary biometric traits, i.e., fingerprints, palmprints, face and iris, but also automate the matching of soft biometric traits, i.e., scars, marks, and tattoos (SMT). This highlights the awareness and importance of utilizing ancillary information in recognizing an individual. There are many situations where primary biometric traits are either corrupted or no longer available, and the soft biometric information is the only available clue to identify a person.

Soft biometric traits are characteristics that provide some identifying information about an individual, but lack the distinctiveness and permanence to sufficiently differentiate any two individuals [5]. Examples of soft biometrics traits include a person's height, weight, gender, eye color, ethnicity, and SMT [5]. Although these soft biometric traits are not sufficient to uniquely differentiate a person, they do contain certain information about the person to help narrow down his identity. This is the reason that many law enforcement agencies collect and maintain this information in their databases. As an example, the FBI booking card includes a suspect's demographic information, such as height, weight, age, gender, etc. in addition to the primary biometric information, i.e., fingerprints. In particular, the booking card also includes prominent scars, marks, and tattoos if present on a subject.

Scars, marks, and tattoos (SMT) are imprints on skin that have been shown to be useful by law enforcement agencies for identification of a non-skeletalized body of a victim or a suspect using a false identity. SMT provide more discriminative information than the traditional demographic indicators such as age, height, gender, race, and gender to identify a person. People get tattoos in order to be identified as distinct from others, to display their personality, 


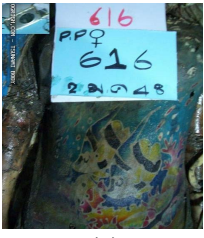

(a)

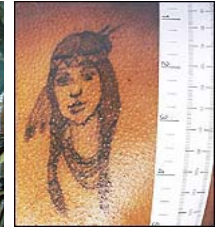

(b)

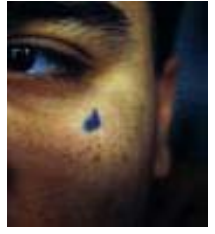

(c)

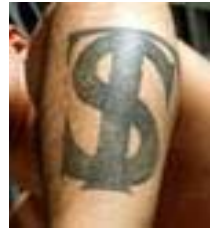

(d)
Fig. 1. Examples of tattoo used for victim and suspect identification: Tattoos on (a) an Asian Tsunami victim and (b) unidentified murdered woman; (c) a prison tattoo (teardrop) and (d) Texas-syndicate gang tattoo

or to exhibit a membership in a group. Hence, presence of tattoos often leads to more comprehensive understanding of the person's background and beliefs.

\subsection{Tattoos}

People have used tattoos for over 5,000 years to differentiate themselves from others [6]. Until recently, the practice of tattooing was limited to particular groups, such as motor bikers, sailors, and members of criminal gangs. But, now tattoos are no longer associated with such unsavory reputations and, as a result, the size of the tattooed population is rising rapidly. The rising popularity of tattoos amongst the younger section of the population is even more surprising. A study published in the Journal of the American Academy of Dermatology in 2006 reported that about 36\% of Americans in the age group 18 to 29 have at least one tattoo [7].

Tattoos engraved on the human body have been successfully used to assist in human identification in forensics applications (see Fig. 1). This is not only because of the increasing prevalence of tattoos, but also due to their impact on other methods of human identification such as visual, pathological, or trauma-based identification [8]. Tattoo pigments are embedded in the skin to such a depth that even severe skin burns often do not destroy a tattoo; tattoos were used to identify victims of the 9/11 terrorist attacks [9] and the Asian tsunami in 2004 [10]. Criminal identification is another important application, because tattoos often contain hidden meaning related to a suspect's criminal history, such as gang membership, religious beliefs, previous convictions, years spent in jail, etc. [11] (see Fig. 2). In addition, a study by Burma [12] suggested that delinquents are significantly more likely to have tattoos than non-delinquents.

Law enforcement agencies routinely photograph and catalog tattoo patterns for the purpose of identifying victims and convicts (who often use aliases). The ANSI/NIST ITL 1-2000 standard [13] defines eight major class labels, e.g., human face, animal, and symbols, and 80 subclass labels for categorizing tattoos (Figures 3 and 4). A search involves matching the class label of a query tattoo with labels associated with tattoos in a database [14]. This tattoo matching process based on human-assigned class labels is subjective, has limited performance, and is very timeconsuming because of the following characteristics of tattoo images. (i) Tattoo images are often composed in terms of multiple objects and cannot be classified into simple categories contained in the ANSI/NIST standard, (ii) a class label (e.g., "flag") does not capture the detailed semantic information available in tattoo images, (iii) tattoo images have large intra-class variability (see Fig. 5), and (iv) the classes defined in the ANSI/NIST standard are not adequate to describe an ever-increasing variety of new tattoo designs.

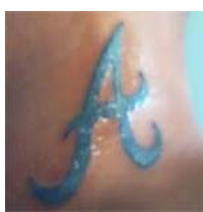

(a)

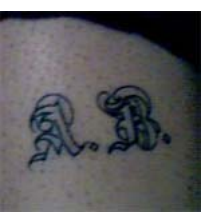

(b)

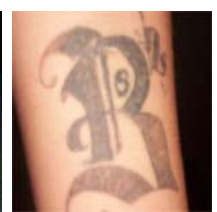

(c)

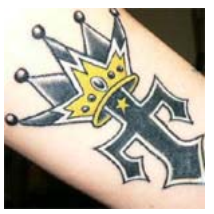

(d)
Fig. 2. Examples of well-known gang tattoos: (a) Ambrose, (b) Adidas boys, (c) Brazers, and (d) Latin kings [11]

\section{CONTENT-BASED TATTOO IMAGE RETRIEVAL}

Due to advances in sensors, storage capabilities, and Web technologies, there has been a tremendous growth in the number of digital images that are readily accessible to consumers and organizations. Flickr, a well-known photo sharing website, reported that there are more than one million new images uploaded to it every day [15]. Users are becoming increasingly interested in capturing and searching for images because of the widespread use of digital cameras and the availability of image retrieval systems, such as Google Image and Yahoo! Image Search [16,17].

Early image retrieval techniques were based on the textual annotation of images, similar to what is practiced now by the law enforcement agencies for retrieving tattoo images. In this approach, images are first annotated with textual keyword(s) and then retrieved using text-based search methods [18]. However, since automatically generating keywords for a wide spectrum of images is not yet feasible, most text-based image retrieval systems require manual annotation of images. This manual annotation is cumbersome, expensive, subjective, and incomplete, especially for large databases. Further, a list of keywords has to be agreed upon before tagging the images as done in the ANSI/NIST-ITL 1-2000 standard for tattoo images mentioned earlier. These shortcomings led to the development of Content-based image retrieval (CBIR) techniques to simplify and improve both the accuracy and speed of image retrieval.

The goal of CBIR is to extract the visual content of an image such as its color, texture, shape of the objects contained in it, and spatial layout to represent and index the image. No explicit keywords are assigned to the image. It is widely recognized that such descriptors in terms of image attributes are more salient and intuitive to express the visual 


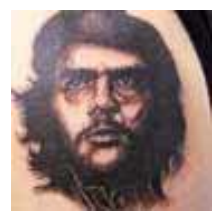

(a)

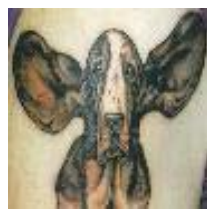

(b)

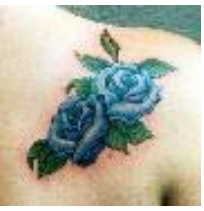

(c)

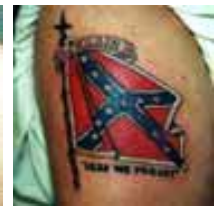

(d)

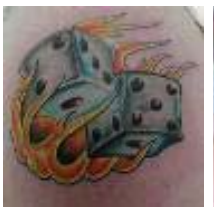

(e)

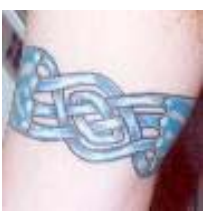

(f)

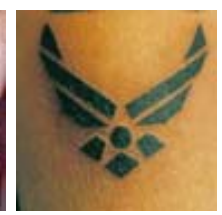

(g)

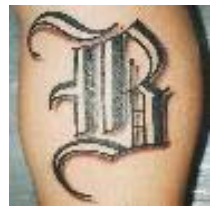

(h)

Fig. 3. Sample tattoos from the eight major classes in the ANSI/NIST ITL 1-2000 standard (a) Human, (b) Animal, (c) Plant, (d)

Flag, (e) Object, (f) Abstract, (g) Symbol, and (h) Other

\begin{tabular}{|c|c|c|c|}
\hline \multicolumn{2}{|c|}{ ANSI/NIST ITL 1-2000 Tattoo Classes } & \multirow{2}{*}{\multicolumn{2}{|c|}{ ANSI/NIST ITL 1-2000 Animal Tattoo Subclas }} \\
\hline & & & \\
\hline Human Forms and Features & HUMAN & \multirow{2}{*}{ Subclass } & \multirow{2}{*}{$\begin{array}{l}\text { Subclass } \\
\text { code }\end{array}$} \\
\hline Animals and Animal & ANIMAL & & \\
\hline & & Cats \& Cat Heads & CAT \\
\hline Plants & PLANT & Dogs \& Dog Heads & DOG \\
\hline Flags & FLAG & Other Domestic Animals & DOMESTIC \\
\hline Objects & OBJECT & \multirow{2}{*}{\begin{tabular}{|l|} 
Vicious Animals \\
(Lions, Tigers, etc.)
\end{tabular}} & \multirow[t]{2}{*}{ VICIOUS } \\
\hline Abstractions & ABSTRACT & & \\
\hline $\begin{array}{l}\text { Insignias \& Symbols } \\
\text { Other Images }\end{array}$ & $\begin{array}{l}\text { SYMBOL } \\
\text { OTHER }\end{array}$ & $\begin{array}{l}\text { Horses } \\
\text { (Donkeys, Mules, etc.) }\end{array}$ & HORSE \\
\hline & & Other Wild Animals & WILD \\
\hline & & Snakes & SNAKE \\
\hline & & Dragons & DRAGON \\
\hline & & Birds (Cardinal, Hawk, etc.) & BIRD \\
\hline & & Spiders, Bugs, and Insects & INSECT \\
\hline & & Abstract Animals & ABSTRACT \\
\hline & & Animal Parts & PARTS \\
\hline & & Miscellaneous Animal Forms & MANIMAL \\
\hline
\end{tabular}

Fig. 4. Tattoo classes defined in ANSI/NIST-ITL 1-2000 [13]
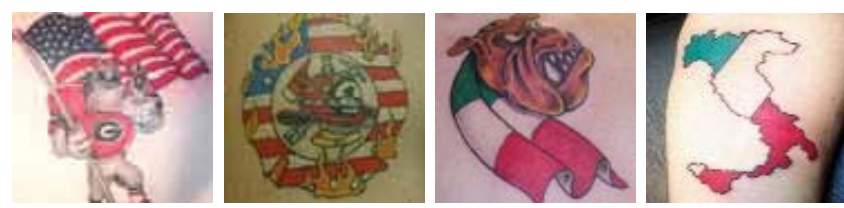

Fig. 5. Illustration of large intra-class variability in tattoo images. All the four images shown here belong to the Flag category

information than simple keywords. However, even though a large number of image retrieval systems have been developed and some are commercially available, the performance of these systems still needs substantial improvement. "Because there is little connection between pixel statistics and the human interpretation of an image (the "semantic gap") the use of large number of generic features makes highly likely that results will not be scalable, i.e. they will not hold on collections of images other than the ones used during the development of the method" [19]. Needless to say, there have been many attempts to compensate for the semantic gap, such as relevance feedback approach [20,21,22,23], learning-based approaches [21,24], salient feature matching [25], composite querying methods [26,27, $28]$ and probabilistic approach $[29,30]$. But the retrieval performance of general purpose CBIR systems is still not very satisfactory.

Compared to the common CBIR formulation, we see the CBIR problem with a different perspective. What if, a user specifically knows what image he is looking for and provides an image as an example? For example, instead of searching for some historical or governmental buildings, the user wants to find different instances (images) of the White House and provides a picture of the White House as a query.
Obviously, the concept of "visually similar" (as opposed to "semantically similar") image retrieval narrows the semantic gap dramatically. This is indeed a well known and active research topic in document and information retrieval community known as known-item retrieval $[31,32,33]$. In this task, the user knows of a particular item that he is looking for, but does not know where it is in the database. This formulation perfectly fits the tattoo image retrieval problem because the goal of the system is to retrieve all visually similar tattoo images.

We present here a CBIR system for tattoo images. Given a query tattoo image, we wish to retrieve all visually similar tattoo images that are in the database. As in other image retrieval systems, top- $\mathrm{N}$ (say, $\mathrm{N}=20$ ) most similar images are retrieved and presented to the user. If the user wishes to see additional retrievals, the system will retrieve the next $\mathrm{N}$ most similar images from the database, and so on. The "user feedback" or "preference" based on retrieved images could be used to improve both the feature extraction as well as the similarity measure used in the matching module. As mentioned earlier, textual information, such as class and subclass labels, is used to annotate and retrieve tattoo images in forensics community. To keep our system compatible with the current practice in law enforcement, a user can specify both the tattoo image and its category information as part of the query (see Fig. 6).

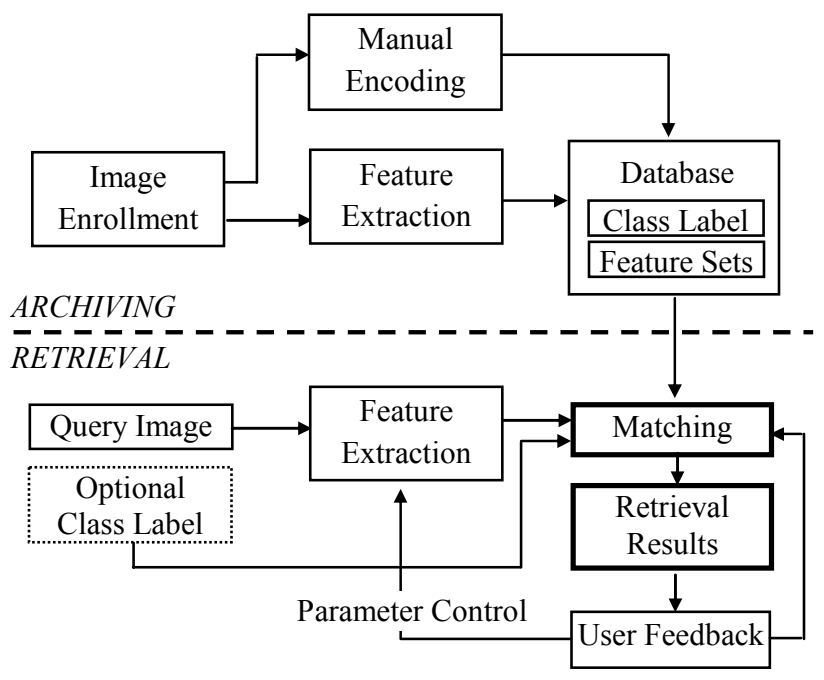

Fig. 6. Tattoo Image Retrieval System 


\section{TATTOO IMAGE RETRIEVAL SYSTEM}

To our knowledge, very little work has been done on CBIR systems for tattoo images. Our previous work used low-level image features (color, shape and texture) to represent and match tattoo images [34]. To improve this system's performance, we designed a rank-based distance metric learning approach [35]. Although metric learning improved the rank-1 retrieval accuracy by $\sim 4 \%$, it is clear that we need to introduce additional features to drastically improve the matching performance. In this paper, we introduce invariant image features based on the Scale Invariant Feature Transform (SIFT) [36] for tattoo image retrieval.

\subsection{SIFT features}

Scale Invariant Feature Transform (SIFT) extracts repeatable characteristic feature points from an image and generates descriptors representing the texture around the feature points [36]. These feature points are invariant to image scale and rotation, and are shown to provide robust matching across a substantial range of affine distortion, change in 3D viewpoint, addition of noise and change in illumination. A brief description of the SIFT operator is provided below and more detailed description of this transform can be found in Lowe [36].

\subsubsection{Scale Space Construction}

The first step of feature points, i.e., keypoint, detection is to identify locations and scales that can be repeatably assigned under differing views of the same object. Finding scale invariant locations is performed by function that searches for stable features across different scales. The scale space of an image is defined as a function, $L(x, y, \sigma)$, that is obtained by convolvung a variable-scale Gaussian, $G(x, y, \sigma)$, with an input image, $I(x, y)$ :

$$
L(x, y, \sigma)=G(x, y, \sigma) * I(x, y)
$$

where $*$ is the convolution operation in $x$ and $y$, and

$$
G(x, y, \sigma)=\frac{1}{2 \pi \sigma} e^{-\left(x^{2}+y^{2}\right) / 2 \sigma^{2}} .
$$

To detect stable keypoint locations in scale space, the difference-of-Gaussian (DoG) function convolved with the image $D(x, y, \sigma)$ is computed from the difference of two nearby scales separated by a constant multiplicative factor $k$ as in

$$
\begin{aligned}
D(x, y, \sigma) & =(G(x, y, k \sigma)-G(x, y, \sigma)) * I(x, y) \\
& =L(x, y, k \sigma)-L(x, y, \sigma) .
\end{aligned}
$$

The DoG function is a close approximation to the scalenormalized Laplacian of Gaussian, $\sigma^{2} \nabla^{2} G$. It is known that the maxima and minima of $\sigma^{2} \nabla^{2} G$ produce the most stable image features compared to a range of other possible image functions, such as the gradient, Hessian, or Harris corner function. The set of Gaussian smoothed images and DoG images is called an octave. A set of such octaves is constructed by successively down sampling the original image. Our system uses four different octaves and six different scales for SIFT.

\subsubsection{Local Extrema Detection}

In order to detect the local maxima and minima of $\mathrm{D}(x, y, \sigma)$, each sample point is compared to its eight neighbors in the current image and nine neighbors in the scale above ad below. It is selected if it is larger than all of the neighbors or smaller than all of them. The cost of this check is reasonably low due to the fact that most sample points will be eliminated following the first few checks.

\subsubsection{Accurate Keypoint Localization}

Once a keypoint candidate has been found by scale-space extrema detection, the next step is to perform a detailed fit to the nearby data for location, scale, and ratio of principal curvatures. This information allows us to reject points that have low contrast (and are therefore sensitive to noise) or poorly localized along an edge. Fig. 7 shows examples of these feature points in three different tattoo images.

\subsubsection{Local Image Descriptor Assignment}

A $16 \times 16$ window is used to generate a histogram of gradient orientation around each local extremum. To make the descriptor rotation invariant, all gradient orientations are rotated with respect to the major orientation of the local extremum.

\subsubsection{Keypoint Matching}

Matching is performed by comparing keypoints in two images based on the associated descriptors using the Euclidean distance metric. We use the number of matching keypoints as the matching score between two images (see Fig. 8).

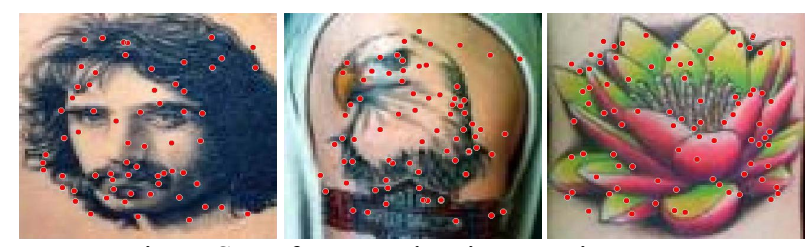

Fig. 7. SIFT feature points in tattoo images

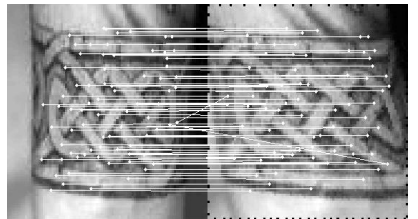

(a) 87 matching points

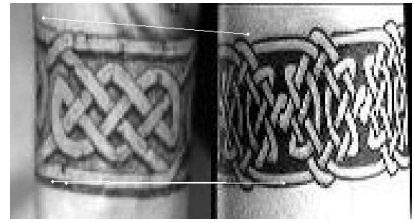

(b) 3 matching points
Fig. 8. Two matching examples with the number of matching key points between a pair of (a) similar and (b) different tattoo images 


\subsection{Tattoo Image Databases}

Two different tattoo image databases were used to evaluate our system. One is a set of tattoo images downloaded from the Web and the other is an operational tattoo image database provided by the Michigan Forensics Department.

\subsubsection{Web-DB: Web-downloaded Tattoo Image Database}

We first tested our system on a set of 4,323 tattoo images downloaded tattoo from the Web. This database is called $W e b-D B,[10,37]$. These $90 \times 90$ color images belong to eight main classes and 30 subclasses as defined in the ANSI/NIST ITL 1-2000 standard [13]. Tattoo images in operational scenarios are often captured under non-ideal conditions (e.g., by a surveillance camera). Hence, multiple acquisition of the same tattoo may appear different because of the imaging conditions, such as brightness, viewpoint, and distance (see examples in Fig. 9). Nevertheless, tattoos in these images are visually similar and a tattoo image retrieval system should be invariant to these imaging conditions. To simulate the realistic imaging conditions, we classify the resulting image transformations into blurring, affine, changes in illumination, color, aspect ratio, and rotation. Figures 10 (b)-(g) show examples of transforming a tattoo image in 11(a). For each tattoo image in the Web-DB database, 20 transformed images are generated by applying transforms with two different intensities of blurring and illumination, four different affine transformations, four different aspect ratios, four different rotations, and four different color changes. This results in a total of 86,460 transformed or synthesized images of 4,323 original tattoo images.

\subsubsection{MI-DB: Michigan State Police Tattoo Database}

Michigan police department has been capturing tattoo images of suspects and convicts for over ten years. Tattoos are photographed when a suspect/convict is booked, is assigned a keyword as defined in the ANSI/NIST standard and then stored in the database along with other demographic information. We were provided with 69,507 operational tattoo images $(640 \times 480$ color images $)$. We call this database $M I-D B$.

We first examined the quality of tattoo images in MI-DB and classified them into three different classes, good, bad, and ugly (same quality designations have been used for latent fingerprints in which are currently used for one of public fingerprint databases, NIST SD27). MI-DB database contains $\sim 65 \%$ good, $\sim 27 \%$ bad, and $\sim 8 \%$ ugly quality tattoo images. The bad or ugly quality of tattoo images is mostly due to the small size of the tattoo or because the tattoo has faded over time. We also that $\sim 20 \%$ of the images in the database are copies of the same tattoo. These copies are present because the same person may have been booked multiple times, or different suspects/convicts have the same tattoo. Because the imaging conditions of those copies are different from each other (see Fig. 9), there is no need to generate the synthetic transforms of the tattoos in this database to simulate different imaging conditions.

In order to use the operational tattoo images, we needed two preprocessing steps, i.e., cropping the tattoo and assigning a keyword to each tattoo. Because the images in the MI-DB contain, in addition to the tattoo, person's clothing, full arm, back torso, etc., cropping the tattoo from the background is necessary. We also manually tagged the tattoo images with keywords defined in the ANSI/NIST standard. The images in the database belong to eight main classes and 80 subclasses as defined in the ANSI/NIST standard. The manual annotating is a time consuming process, our goal here is to demonstrate the advantages of image based matching over keyword-based retrieval. In contrast to practice in law enforcement, where only one keyword is assigned per image, we assign each tattoo with multiple keywords because in many instances, there are indeed multiple "objects" in a tattoo.

\section{EXPERIMENTAL RESULTS}

To evaluate the retrieval performance of our system, one of the copies of the same tattoo is used as a query image to retrieve its visually similar image(s) in the database. For Web-DB, we set up an experimental scenario to mimic the situation where the query tattoo images from victims/suspects are of less than perfect quality (distorted, noisy, blurred and occluded). Because the images in the database are supposed to have been collected under controlled conditions at booking time, they are assumed to be of higher quality. We simulate this low-quality query situation by applying the image transformations to the high quality images downloaded form the web (see Figures 9 and 10). As a result, for the Web-DB, the number of query images is 86,460 and the number of images in the gallery database is 4,323. A retrieved image is deemed to be relevant or correct when the query image was generated from the retrieved image via one of the specified image transformations. On the other hand, because MI-DB already contains many copies of the same tattoo, we use one of the copies as a query image and aim to retrieve other image(s) of the same tattoo. We used 500 queries against 11,000 randomly selected from the MI-DB to perform the retrieval experiment on MI-DB. Since our system aims to retrieve the correct image at the top rank, we adopted the cumulative matching characteristic (CMC) curve [38] as the evaluation metric.

Fig. 11 shows the retrieval performances based on SIFT features proposed here and low-level image features (color, shape, and texture) reported in [34]. Using SIFT features on Web-DB, the rank-1 retrieval accuracy is $\sim 97.1 \%$ and the rank-20 retrieval accuracy is $\sim 98.6 \%$. The corresponding accuracies reported in [34] are $\sim 68.6 \%$ and $\sim 82.5 \%$, respectively. Note that the experiments in [34] were 


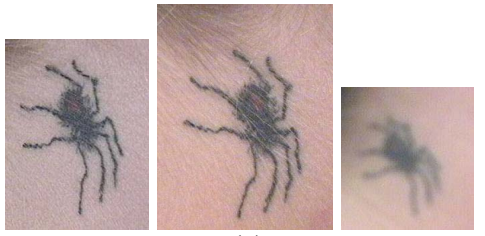

(a)

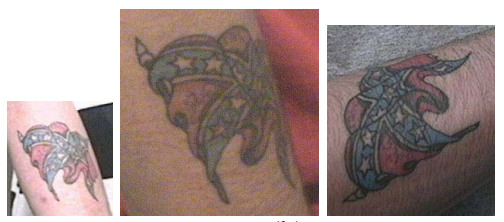

(b)

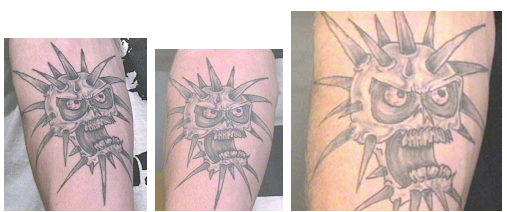

(c)

Fig. 9. Examples of tattoo copy images in the Michigan Police database. Three tattoos each in (a), (b), and (c) are copies of the same tattoo. These tattoo copy images were captured at different times

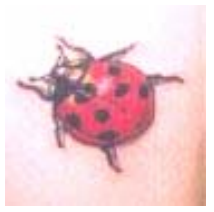

(a)

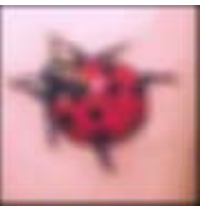

(b)

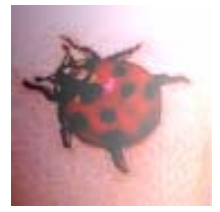

(c)

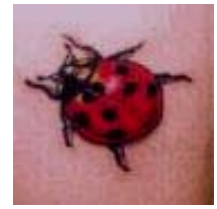

(d)

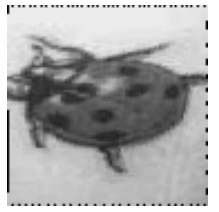

(e)

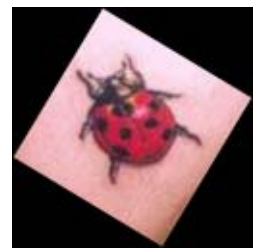

(g)

Fig. 10. Examples of image transformations: (a) original and variations due to (b) blurring, (c) illumination change, (d) color component changes, (e) affine transformation, (f) aspect ratio change, and (g) rotation

conducted on only a subset (approximately half) of the database used here. This shows that SIFT features are salient and robust in capturing the tattoo image content. SIFT features are particularly effective in processing query images that are blurred and have uneven illumination, which were found to be the most difficult cases for the system in [34]. For example, only $67 \%$ of blurred and $42 \%$ of illumination change queries appeared in top-20 retrievals in [34], but using SIFT features, these accuracies were increased to $94 \%$ and $92 \%$, respectively. Only severely transformed images that were hardly recognizable to a human eye as a tattoo were not correctly retrieved (see Fig. 12).

While SIFT feature showed high retrieval accuracy on Web-DB, its substantially lower retrieval performance on MI-DB, $\sim 31.9 \%$ at rank- 1 and $\sim 77.2 \%$ at rank-20, reveals limitations of current approach in using SIFT feature. First, current matching produces many false matches if there is a big size difference between the two images. Because the number of extracted keypoints is proportional to the image size, the difference in the numbers of keypoints extracted between two very different sized images could be rather large. The possibility of false matches in this case is, therefore, much higher than that of between two similar sized images. Further, SIFT-based matching allows multiple matches, i.e., several keypoints in a query image could be matched to a single keypoint in the gallery image, leading to many false matches (see Fig. 13.). There is also large variance in image size in the MIDB, which makes the retrieval problem difficult using SIFT feature directly.

\section{CONCLUSIONS AND FUTURE WORK}

We have presented a CBIR system for tattoo images. With the growing use of tattoos for victim and suspect identification in forensics and law enforcement agencies, such a system will be of great value in apprehending suspects and identifying victims. We introduced image similarity computation based on SIFT features. Our experiment results show rank-20 retrieval accuracies of $98.6 \%$ on a good quality database (Web-DB) and $77.2 \%$ on an operational database (MI-DB). For Web-DB, the average execution time for feature extraction is 0.0005 sec/image and the average matching time between an image pair is $0.005 \mathrm{sec}$ on Intel Core 2, $2.66 \mathrm{GHz}, 3 \mathrm{~GB}$ RAM processor. Since the images in MI-DB are usually much bigger than in Web-DB, feature extraction $(0.0023$ sec/image), and matching ( $0.3255 \mathrm{sec} /$ image pair) take longer time on the same machine.

Although the difference between the number of matching keypoints between rank 1 and rank 2 retrieved images is quite large in the case of Web-DB $(\sim 34)$, this difference is very small for the MI-DB $(\sim 11)$. This indicates a need to utilize additional features and refine our similarity measure to handle difficult and challenging images encountered in an operational database. We plan to introduce some constraints among the matching points to delete many of the false multiple matchings. Further, we will utilize color information in the neighborhood of matching points to improve the retrieval performance.

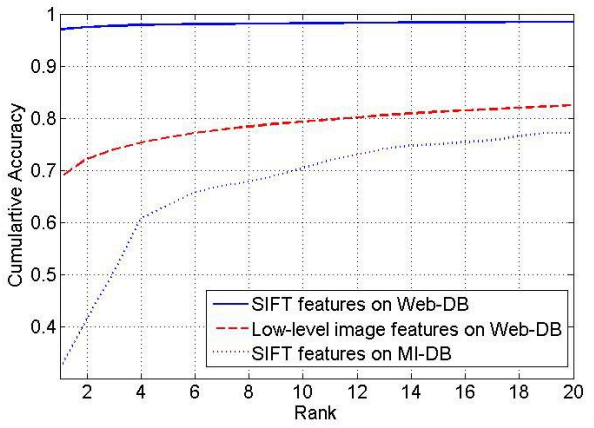

Fig. 11. CMC curves comparing the retrieval performances based on SIFT features (Web-DB and MI-DB) and low-level image features (Web-DB only) 


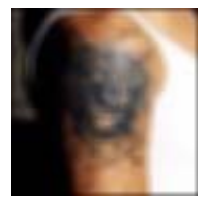

(a)

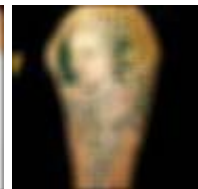

(b)

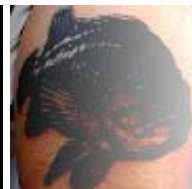

(c)

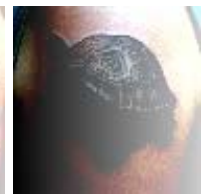

(d)
Fig. 12. Four queries in MI-DB for which the top-20 retrieved image did not contain the correct tattoo image. The correct tattoo image is found at ranks (a) 30, (b) 151, (c) 122 and (d) 665

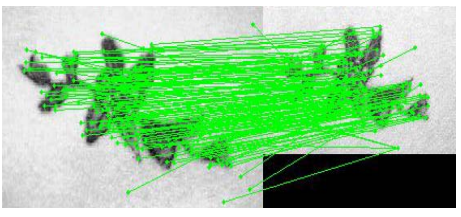

(a) 138

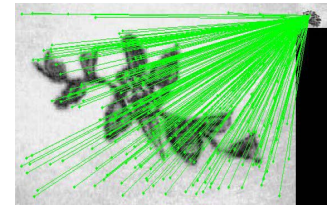

(b) 107
Fig. 13. Examples of SIFT keypoint matching between (a) two visually similar tattoos with the same size and (b) two different tattoos with large size difference in MI-DB database. Number of matching keypoints is shown

\section{ACKNOWLEDGEMNET}

The authors would like to thank Scott Swan, FBI and Lt. Gregory Michaud, Michigan Forensics Laboratory for their encouragement and assistance. This research was supported by NSF IUC on Identification Technology Research (CITeR) and Army Research Office.

\section{REFERENCES}

[1] A. K. Jain, P. Flynn, and A. Ross (eds.), Handbook of Biometrics, Springer, 2007.

[2] Biometrics Overview, http://www.biometrics.gov/ Document s/BioOverview.pdf

[3] Fingerprint Vendor Technology Evaluation, http://fpvte.nist .gov/

[4] Press Release, FBI, http://www.fbi.gov/pressrel/pressrel08/ ngicontract021208.htm

[5] A.K. Jain, S.C. Dass and K. Nandakumar. Can soft biometric traits assist user recognition? In Proc. SPIE Conf. Biometric Technology for Human Identification, 2004

[6] A Brief History of Tattoos, http://www.designboom.com/his tory/tattoo_history.html

[7] Tattoo Facts and Statistics, http://www.vanishingtattoo.com /tattoo_facts.htm, Oct. 2006,

[8] T. Thompson and S. Black. Forensic Human Identification, An Introduction. CRC Press, 2007.
[9] E. Lipton, and J. Glanz. Limits of DNA Research Pushed to Identify the Dead of Sept. 11, NY Times, Apr. 22, 2002

[10] Decay challenges forensic skills, The Standard-Times, Jan. 8. 2005.

[11] Gang Ink, http://www.gankink.com/

[12] J. H. Burma. Self-tattooing among delinquents: A research note. Sociology and Social Research, Vol. 43, pp. 341-345, 1959.

[13] ANSI/NIST-ITL 1-2000, Data Format for the Interchange of Fingerprint, Facial, \& Scar Mark \& Tattoo (SMT),

[14] GangNet: A 21 $1^{\text {st }}$ Century Solution to the Gang Problem, Dec. 2006 http://psd.orionsci.com/Products/Gangnet.asp

[15] Geotagging - one day later http://blog.flickr.com/flickrblog /2006/08/geotagging_one_html (August 29, 2006)

[16] Google Image, http://images.google.com/imghp

[17] Yahoo Image, http://images.search.yahoo.com/

[18] A. M. W. Smeulders, M. Worring, S. Santini, A. Gupta, and R. Jain, Content-based image retrieval at the end of the early years, IEEE Trans. PAMI, Vol.22, pp. 1349-1380, 2000.

[19] T. Pavlidis, Limitations of Content-based image retrieval http://www.theopavlidis.com/technology/CBIR/PaperB/vers3.ht $\mathrm{m}$

[20] Y. Rui, T. Huang, and S. Mehrotra, Content-based image retrieval with relevance feedback in MARS. In Proc. ICIP, Vol. 2, pp. 815-818, 1997

[21] Y. Wu, Q. Tian, and T. S. Huang, Discriminant EM algorithm with application to image retrieval. In Proc. CVPR, Vol. 1, pp. 222-227, 2000

[22] Y. Chen, and J. Z. Wang, A region-based fuzzy feature matching approach to content-based image retrieval, IEEE Trans. PAMI, Vol.24, pp. 252-1267, 2002.

[23] Y. Rui, and T. S. Huang, Optimizing learning in image retrieval. In Proc. CVPR, Vol. 1 pp. 236-243, 2000.

[24] M. Webe, M. Welling, and P. Perona, Unsupervised learning of models for recognition. In Proc. ECCV, pp. 18-32, 2000.

[25] H. Wolfson, and I. Rigoutos, Geometric hashing: An overview. IEEE Trans. Computer Science \& Engineering, Vol. 4, pp. 10-21, 1997.

[26] A. Chalechale, G. Naghdy, and A. Mertins, Sketch-based image matching using angular partitioning. IEEE Trans. Systems, Man and Cybernetics, Vol. 35, pp. 28-41, 2005 


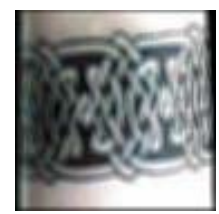

Query \#1

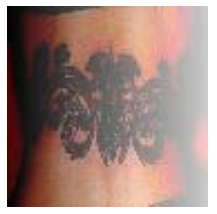

Query \#2

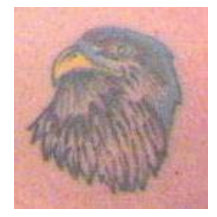

Query \#3

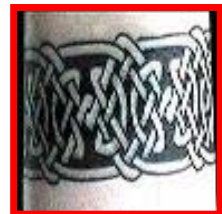

59

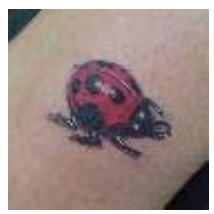

12

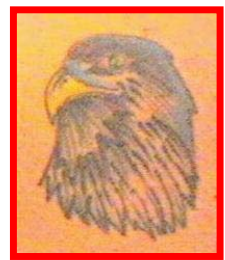

110

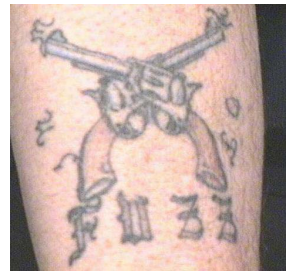

Query \#4
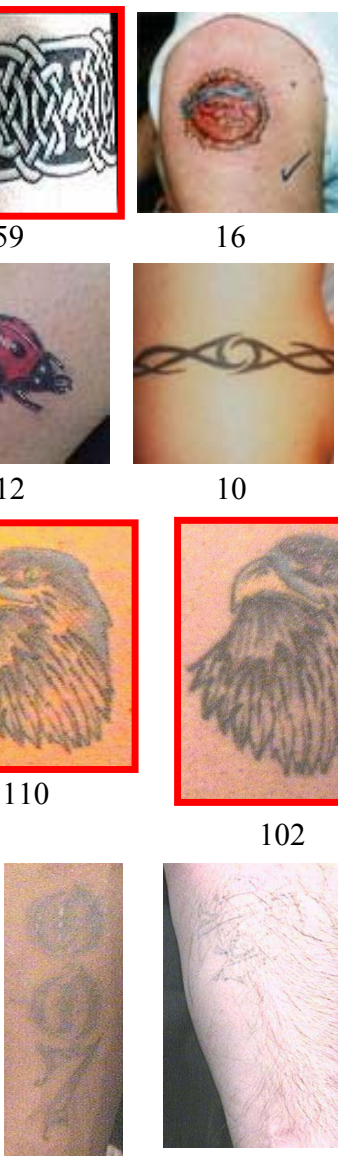

68
16

10

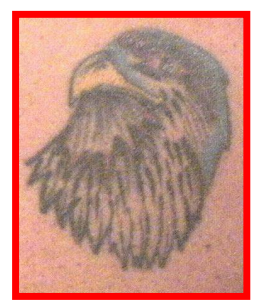

102
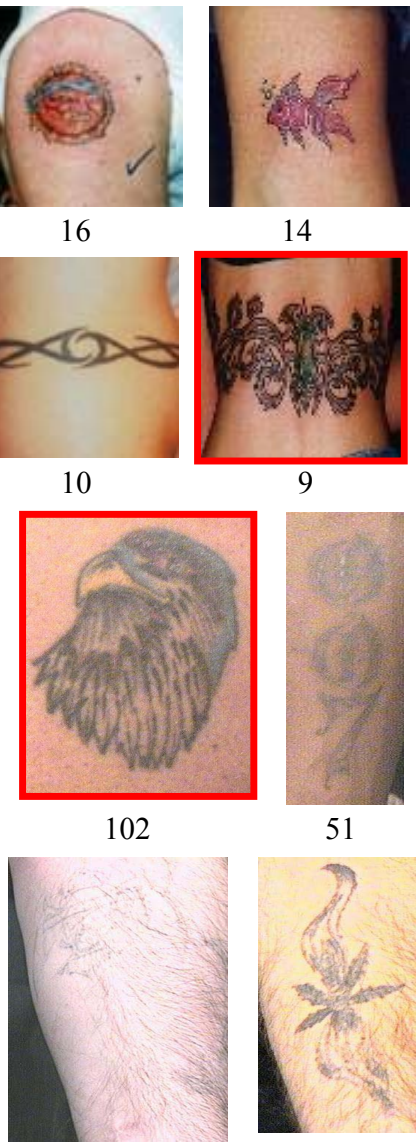

67
14

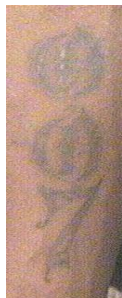

51
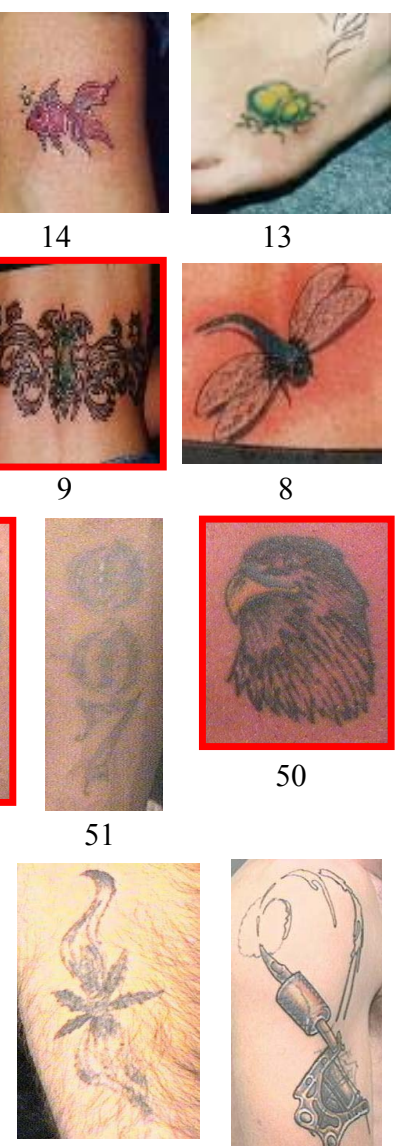

59
13

8

50

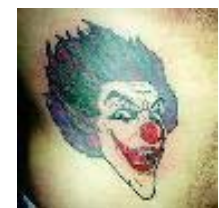

12

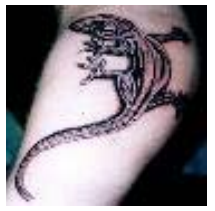

8

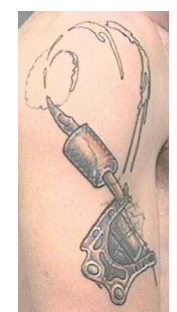

47

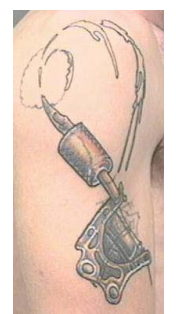

54

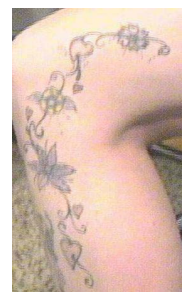

51

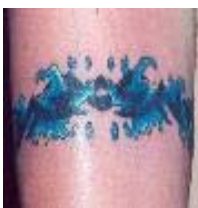

11

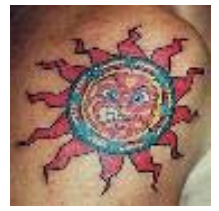

8

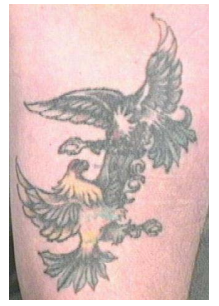

47

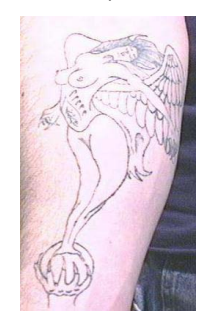

50

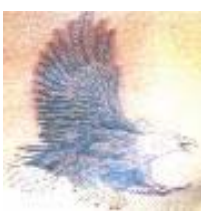

11

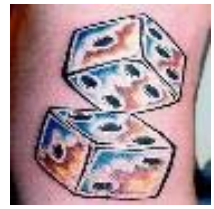

8

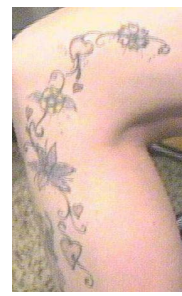

43

Fig. 14. Examples of retrieval experiments on Web-DB (the first and second rows) and MI-DB (the third and fourth rows). Each example contains a query image and its top-7 retrieved images with the number of matching keypoints.

[27] J. Assfalg, A. Del Bimbo, and P. Pala, Three-Dimensional interfaces for querying by example in content-based image retrieval. IEEE Trans. Visualization and Computer Graphics, Vol. 8, pp. 305-318, 2002

[28] T. Kaster, M. Pfeiffer, and C. Bauckhage, Combining speech and haptics for intuitive and efficient navigation through image databases. In Proc. ICMI, pp. 180-187, 2003

[29] R. Jin, and A. Hauptmann, Using a probabilistic source model for comparing images. In Proc. ICIP, Vol. 3, pp. 941-944, 2002

[30] N. Vasconcelos, and A. Lippman, A probabilistic architecture for content-based image retrieval. In Proc. CVPR, Vol. 1, pp. 216221,2000 .

[31] P. Ogilvie, and J. Callan, Combining document representations for known-item search. In Proc. Int. Conf. on Research and Development in Information Retrieval, pp. 143-150, 2003.
[32] J. Kamps, G. Mishne, and M. de Rijke, Language models for searching in Web corpora, TREC 2004, NIST Special Publication, pp.500-561, 2005.

[33] S. Yahyaei, and C. Monz, Applying Maximum Entropy to Known-Item Email Retrieval, In Proc. European Conf. on Information Retrieval, pp. 406-413, 2008

[34] A. K. Jain, J.-E. Lee, and R. Jin, Tattoo-ID: Automatic tattoo image retrieval for suspect \& victim identification. In Proc. Pacific-Rim Conf. on Multimedia, pp. 256-265, 2007.

[35] J.-E. Lee, R. Jin, and A. K. Jain, Rank-based distance metric learning: An application to image retrieval. In Proc, CVPR, 2008

[36] D. Lowe, Distinctive image features from scale-invariant keypoints, IJCV, Vol. 60, Issue 2, pp. 91-110, 2004.

[37] Online Tattoo Designs, http://www.tattoodesing.com/gallery/

[38] H. Moon, and P. J. Phillips, Computational and Performance aspects of PCA-based Face Recognition Algorithms, Perception, Vol. 30, pp. 303-321, 2001 